\title{
Arthropathy-like findings and a carpal tunnel syndrome as the presenting features of Scheie syndrome: Three cases from the same family
}

\author{
Songül Gökay ${ }^{1}$, Fatih Kardaş ${ }^{1}$, Mustafa Kendirci ${ }^{1}$, Betül Sözeri ${ }^{2}$ \\ Divisions of ${ }^{1}$ Pediatric Nutrition and Metabolism and ${ }^{2}$ Pediatric Rheumatology, Department of Pediatrics, Faculty of \\ Medicine, Erciyes University, Kayseri, Turkey. \\ E-mail: songulsa@yahoo.com \\ Received: 17th April 2017, Revised: 9th June 2017, Accepted: 10th June 2017
}

SUMMARY: Gökay S, Kardaş F, Kendirci M, Sözeri B. Arthropathy-like findings and a carpal tunnel syndrome as the presenting features of Scheie syndrome: Three cases from the same family. Turk J Pediatr 2018; 60: 344-347.

Mucopolysaccharidosis (MPS) type $I$ is a rare autosomal recessive disease caused by a deficiency of the lysosomal enzyme $\alpha$-L-iduronidase. MPS I is divided into three subtypes based on the severity of symptoms: Hurler, Hurler-Scheie, and Scheie syndrome (severe, intermediate, and mild forms, respectively). Musculoskeletal involvement may be the only presenting sign in the patients with Scheie syndrome. We have reviewed three cases with prominent features of carpal tunnel syndrome (CTS) at the onset of their disease. Diagnosis was delayed in almost all cases (range 16-19 years). During one year of follow-up period, alleviations of the pain in the hands of patients were observed after enzyme replacement therapy. MPS type I should be considered in the differential diagnosis of the patients with CTS in the first and second decades of life, particularly with stiffness of the fingers and difficulty using the hands without an inflammatory component. An increased awareness of the disease may contribute to more accurate diagnosis, and patients may benefit from early intervention.

Key words: arthropathy, carpal tunnel syndrome, mucopolysaccharidosis, Scheie syndrome.

Mucopolysaccharidosis type I (MPS I) is an autosomal recessive disorder resulting from decreased activity of the lysosomal enzyme $\alpha$-L-iduronidase (IDUA). Accumulation of glycosaminoglycans (GAGs) in the lysosomes causes a progressive multisystem disease.

It is divided into three subtypes based on the severity of disorders: Hurler, Hurler-Scheie, and Scheie syndrome. Scheie syndrome is one of the less severe form of MPS $\mathrm{I}^{1}{ }^{1}$ A definitive diagnosis of the Scheie syndrome may be delayed for years since early symptoms are difficult to recognize by physicians who are not familiar with the disease.

\section{Case Reports}

An 18-year-old female patient, her 16-yearold brother and their 19-year-old male cousin born to consanguineous parents, were referred by rheumatologist to our division with the complaints of pain, movement limitations. Siblings have had pain and deformities of the hands for about nine and seven years, respectively. They have applied to pediatrics, orthopedics and physical therapy specialists many times before, but not diagnosed. Medical history revealed that sister suffered and operated because of the unilateral carpal tunnel syndrome (CTS) two years ago. On physical examination, siblings appeared with claw hands with flexion contractures (Fig. 1-2), but their cousin was normal. Systemic evaluations of the patients were unremarkable. Their appearances were normal: no coarse facial appearance or evidence of growth failure or no evidence of any other joint involvement

The cases were presented as poster at the 5th International Congress of Lysosomal Diseases, 14-17 April 2016, Bodrum, Turkey. 

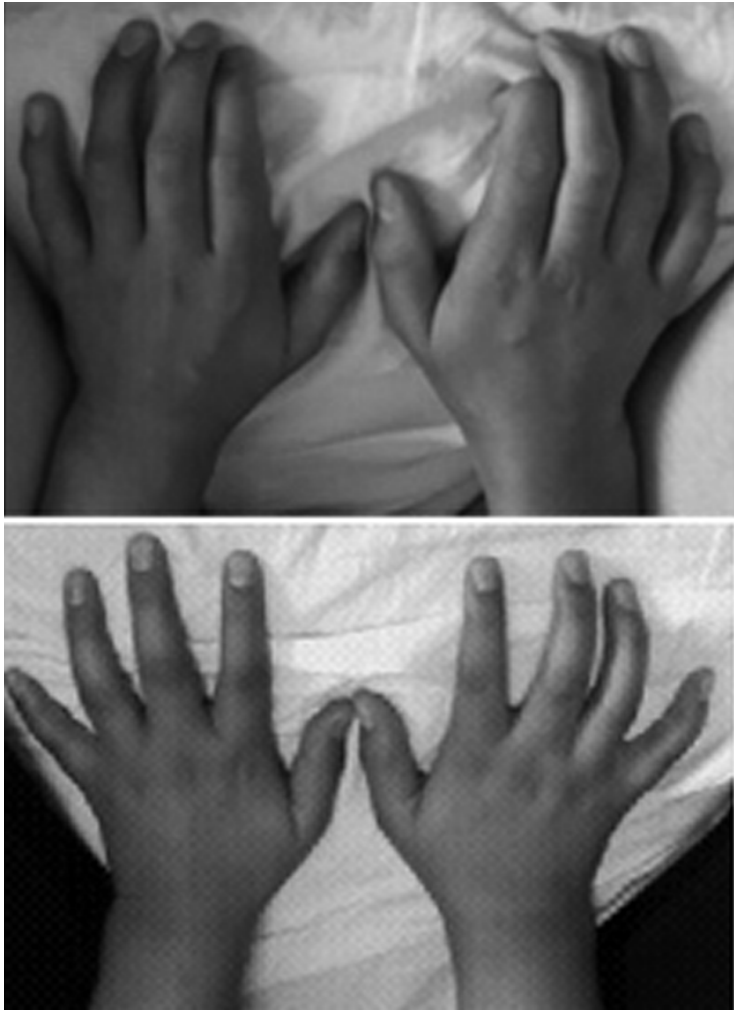

Fig. 1. The appearance of claw hands of case 1 (left) and case 2 (right).

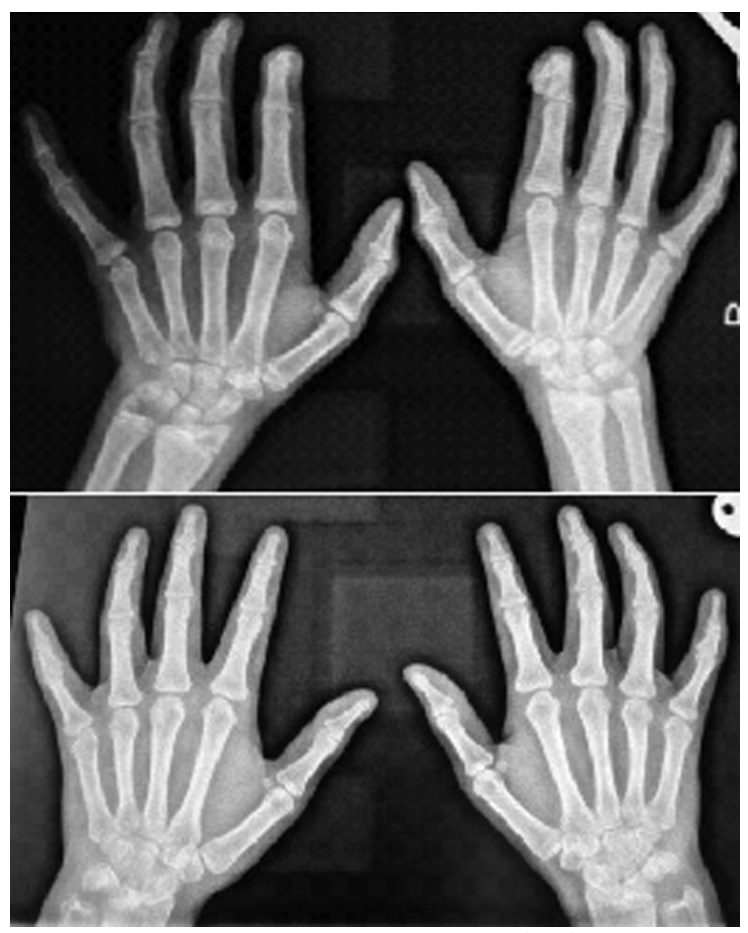

Fig. 2. X-ray of the hands of case 1 (left) and case 2 (right) showing flexion contractures.

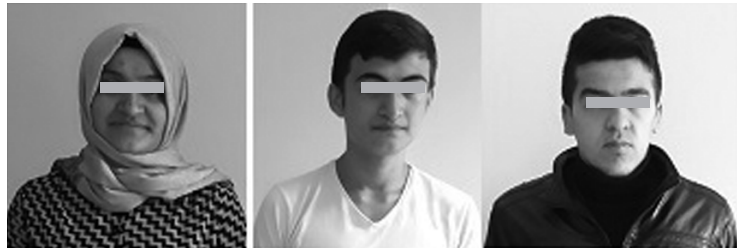

Fig. 3. The facial appearances of case 1 (left), case 2 (in the middle) and case 3 (right).

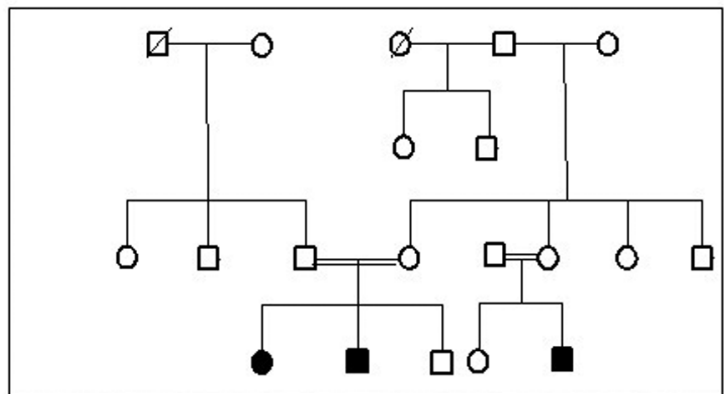

Fig. 4. Pedigree of the family. Squares and circles indicate male and female members, respectively. Heavy lines indicate consanguineous marriages. Black filled squares and circles indicate those individuals who are affected with Scheie syndrome.

(Fig. 3). They had normal intelligence. In the radiological evaluation, there was no evidence of dysostosis multiplex of any other bones. The ophthalmological examinations, hearing tests, echocardiography, intelligence tests and brain magnetic resonance imaging (MRI) of the patients were normal. Electroneurography showed bilaterally signs of severe median nerve suffering for sister and mild for brother and their cousin. Clinical and laboratory characteristics of the patients were summarized in Table I.

IDUA activities were $0.36 \mu \mathrm{mol} / \mathrm{g} / \mathrm{h}, 0.64$ $\mu \mathrm{mol} / \mathrm{g} / \mathrm{h}$ and $0.57 \mu \mathrm{mol} / \mathrm{g} / \mathrm{h}$, respectively (normal range, 10-50). We detected homozygous frame shift mutation c.(521delA); (521delA) for sister and homozygous mutation c. $(1189+4 \mathrm{~A}>\mathrm{T}) ;(1189+4 \mathrm{~A}>\mathrm{T})$ for brother and their cousin in the IDUA gene. Based on the history and physical examination findings there was a marked deficiency of IDUA activity and mutation analysis consistent with the diagnosis of MPS I (Scheie phenotype). Physiotherapy and rehabilitation therapy were programmed for them to use their hands easily in daily activities. In addition, the parents were given genetic counseling. 


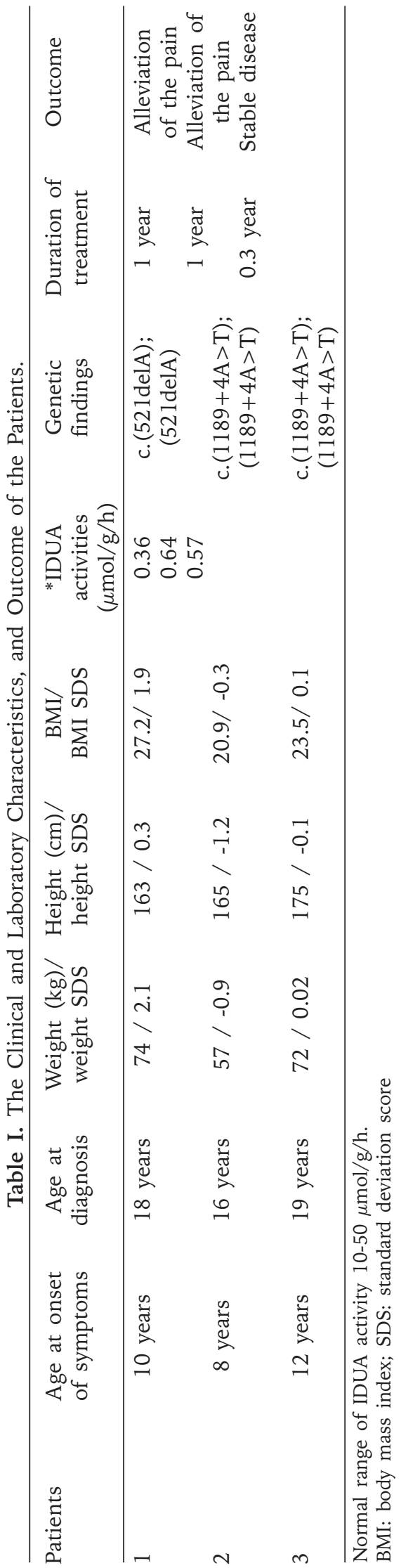

These three cases were started on enzyme replacement therapy (ERT) with laronidase, recombinant human IDUA. Siblings are treated for 12 months and their cousin is treated for four months. Clinical follow-up did not show any progression after 12 months of ERT therapy for siblings. Alleviation of the pain in the hand of siblings was observed; there was no improvement of joint range of motion. An informed consent was received from the family.

\section{Discussion}

MPS I is a rare, progressive, life-threatening autosomal recessive condition caused by defects in the gene coding the IDUA enzyme. Reduced levels of the IDUA enzyme result in impaired lysosomal degradation and accumulation of the GAGs, which leads to progressive cellular and multi-organ dysfunction with variable phenotypes. ${ }^{1}$

Clinical subtypes of MPS I are differentiated by the presence or absence of CNS involvement, the rate of disease progression, the severity of visceral manifestations include coarsening facial features, corneal clouding, hepatomegaly, bone involvement, inguinal or umbilical hernias, and the age of onset. ${ }^{2}$ The mild subtypeScheie phenotype has a more attenuated disease course but can experience a range of progressively debilitating manifestations. In Scheie syndrome, symptoms generally begin to appear after 5 years of age, but the diagnosis is most commonly made after 10 years of age because of the absence or mildness of clinical signs. It has been shown that cases of Scheie syndrome are misdiagnosed as other diseases or undiagnosed for long periods of time (4-54 years). ${ }^{3}$ Early symptoms in the Scheie phenotype include joint contractures, hernias, and carpal tunnel syndrome. Patients with this phenotype show normal intellectual development, can attain normal stature, and usually survive into adulthood, although often with significant morbidity. ${ }^{4}$

Especially contractures of the distal interphalangeal joints including claw hand deformity frequently occur in Scheie syndrome. Joint contractures were reported in $87.7 \%$ of patients in a series of 78 cases of Scheie syndrome by Thomas et al. ${ }^{5}$ In our cases, the first complaints were pain in the hands, thereafter contracture has occurred in the 
hands of the siblings. Although patients were subsequently diagnosed with $\mathrm{CTS}^{6}$, the underlying disease was missed in the patients of Scheie syndrome just as our first case. Scheie syndrome may be misdiagnosed by the inexperienced practitioners as other musculoskeletal and rheumatologic diseases (eg. spondyloarthropathies and juvenile idiopathic arthritis). The lack of inflammatory signs of joint contracture may easily differentiate Scheie syndrome from rheumatologic disorders. ${ }^{3}$

Definitive diagnosis of MPS I is made by enzyme assay demonstrating deficient IDUA activity and can be confirmed by genotyping. ${ }^{7}$ In this report homozygous mutation of c.(521delA); (521delA) for sister could not found in the literature and database search like HGMDPublic. In silico analysis done with Mutation Taster, PolyPhen ${ }^{2}$, SIFT and PROVEAN programs predicted this frame shift mutation as a disease causing variant. As the location of this single base substitution is conserved in different species, it is evaluated as a "likely pathogenic" variant. Splice site mutation of c. $(1189+4 \mathrm{~A}>\mathrm{T}) ;(1189+4 \mathrm{~A}>\mathrm{T})$ for brother and his cousin have already been reported in MPS I by Bertola F, et al. ${ }^{8}$

Recently, ERT with recombinant human IDUA has been shown to be safe and effective in some aspects for patients with the milder HurlerScheie and Scheie phenotypes. ${ }^{9}$ Gabrielli O, et al. ${ }^{10}$ reported clinical and laboratory results in a 12-year-old patient affected by the attenuated form of MPS I treated by ERT from the age of 5 months and compared with his 17-yearold affected sister, who started therapy at 5 years of age. It appears that initiation of ERT when significant arthropathy is present leads to some improvement of joint range of motion in the first 1-2 years of treatment, followed by disease stabilization or a slower rate of disease progression. These observations have shown that the early diagnosis is essential for successful treatment before occurrence of irreversible organ disorders. ${ }^{10}$ There was no progression in our cases during the clinical follow-up after ERT therapy. Alleviation of the pain was observed in the hand of all cases. But there was no improvement of joint range of motion in the siblings. In assessing response to ERT, it requires a longer time.

Although there is widespread symptomatology, diagnosis of Scheie syndrome may be challenging because patient's appearance can be completely normal, and arthropathy-like findings may be the initial symptoms. CTS may be the only clue of diagnosis in this group of patients. Recognition of key symptoms and early diagnosis is the only way to prevent possible complications for this rare but treatable disease.

\section{REFERENCES}

1. Beck M, Arn P, Giugliani R, et al. The natural history of MPS I: Global perspectives from the MPS I Registry. Genet Med 2014; 16: 759-765.

2. Meikle PJ, Hopwood JJ, Clague AE, Carey WF. Prevalence of lysosomal storage disorders. JAMA 1999; 281: 249-254.

3. Cimaz R, Vijay S, Haase C, et al. Attenuated type I mucopolysaccharidosis in the different diagnosis of juvenile idiopathic arthritis: A series of 13 patients with Scheie syndrome. Clin Exp Rheumatol 2006; 24: 196-202.

4. Bahadir C, Kurtulus D, Cihandide E. Mucopolysaccharidosis type-IS presenting with onset of carpal tunnel syndrome at adolescence. J Clin Rheumatol 2009; 15: 402-404.

5. Thomas JA, Beck M, Clarke JT, Cox GF. Childhood onset of Scheie syndrome, the attenuated form of mucopolysaccharidosis I. J Inherit Metab Dis. 2010; 33: 421-427.

6. Haddad FS, Jones DH, Vellodi A, Kane N, Pitt MC Carpal tunnel syndrome in the mucopolysaccharidoses and mucolipidoses. J Bone Joint Surg Br 1997; 79: 576-582.

7. Hall CW, Liebaers I, Di Natale P, Neufeld EF. Enzymatic diagnosis of the genetic mucopolysaccharide storage disorders. Methods Enzymol 1978; 50: 439-456.

8. Bertola F, Filocamo M, Casati G, et al. IDUA mutational profiling of a cohort of 102 European patients with mucopolysaccharidosis type I: identification and characterization of 35 novel $\alpha$-L-iduronidase (IDUA) alleles. Hum Mutat 2011; 32: 2189-2210.

9. Muenzer J, Wraith JE, Clarke LA. Mucopolysaccharidosis I: Management and treatment guidelines. Pediatrics 2009; 123: 19-29.

10. Gabrielli O, Clarke LA, Ficcadenti A, et al.12 year follow up of enzyme-replacement therapy in two siblings with attenuated mucopolysaccharidosis I: The important role of early treatment. BMC Med Genet 2016; 17: 19 